\title{
EFICIÊNCIA DE ÍNDICES FENOTÍPICOS DE COMPRIMENTO DE RAIZ SEMINAL NA AVALIAÇÃO DE PLANTAS INDIVIDUAIS DE MILHO QUANTO Ȧ TOLERÂNCIA AO ALUMÍNIO' ${ }^{1}$
}

\author{
PAULO ROBERTO MARTINS ${ }^{2}$, SIDNEY NETTO PARENTONI ${ }^{3}$, MAURÍCIO ANTÔNIO LOPES ${ }^{4}$ e EDILSON PAIVA ${ }^{4}$
}

\begin{abstract}
RESUMO - O objetivo deste trabalho foi testar a eficiência do comprimento relativo de raiz seminal (CRRS) e do comprimento líquido de raiz seminal (CLRS) como indicadores fenotípicos quanto à tolerância ao alumínio na avaliação de plantas individuais de milho. Plântulas de genótipos tolerantes e suscetíveis ao $\mathrm{Al}$ foram submetidas a solução nutritiva contendo nível tóxico deste elemento, por um período de sete dias, após o qual, determinaram-se os valores de CRRS e CLRS. Os resultados obtidos quando se utilizaram valores médios para CRRS e CLRS mostraram que ambos os índices foram capazes de discriminar com eficiência os materiais tolerantes dos suscetíveis. Entretanto, quando foram utilizados os valores de CRRS e CLRS obtidos a partir de plantas individuais, observou-se a existência de plantas tolerantes com valores típicos de plantas suscetíveis, o que indica que a avaliação fenotípica de plantas individuais pelos dois índices está sujeita a erros significativos, principalmente na caracterização de plantas suscetíveis. Portanto, em estudos para mapeamento de "quantitative trait loci" (QTLs) ligados à tolerância ao $\mathrm{Al}$, nos quais utilizam-se estes índices fenotípicos, o mais apropriado é avaliar famílias $\mathrm{F}_{3}$, onde é possível obter valores médios para CRRS e CLRS e utilizar estas médias para representar os valores fenotípicos das respectivas plantas $\mathrm{F}_{2}$.
\end{abstract}

Termos para indexação: solução nutritiva, toxidez de alumínio, avaliação de plântulas.

\author{
EFFICIENCY OF ROOT LENGTH PHENOTYPIC INDEX IN THE EVALUATION \\ OF INDIVIDUAL MAIZE PLANTS FOR ALUMINUM TOLERANCE
}

\begin{abstract}
The objective of this work was to verify the efficiency of the relative seminal root length (RSRL) and net seminal root length (NSRL) as phenotypic indexes for aluminum tolerance in individual maize plants. Seedlings of Al tolerant and susceptible genotypes were grown in nutrient solution containing toxic level of $\mathrm{Al}$ for a period of seven days, after which the values of RSRL and NSRL were determined. The results obtained when mean values of RSRL and NSRL were utilized showed that both indexes were able to discriminate tolerant from susceptible maize genotypes. However, when individual values of RSRL and NSRL were utilized, some tolerant plants scored as susceptible ones were observed, showing that the phenotypic evaluation of individual plants by both indexes is subjected to significant errors, mainly in the characterization of susceptible plants. Therefore, in studies for quantitative traits loci (QTLs) mapping for Al tolerance in maize, where these indexes are used, the most appropriate is to use $\mathrm{F}_{3}$ families, where it is possible to obtain mean values for RSRL and NSRL and then utilize these means to represent the phenotypic values of the respective $F_{2}$ plants.
\end{abstract}

Index terms: nutrient solution, aluminum toxicity, seedlings evaluation

\footnotetext{
${ }^{1}$ Aceito para publicação em 9 de março de 1999.

${ }^{2}$ Eng. Agr., M.Sc., Embrapa-Centro Nacional de Pesquisa de Milho e Sorgo (CNPMS). (In memoriam.)

${ }^{3}$ Eng. Agr., M.Sc., Embrapa-CNPMS. Caixa Postal 151, CEP 35701-970 Sete Lagoas, MG.

${ }^{4}$ Eng. Agr., Ph.D., Embrapa-CNPMS. E-mail: edilson@cnpms.embrapa.br
}

\section{INTRODUÇÃO}

A acidez do solo é reconhecida como uma das principais causas das limitações da cultura do milho nas regiões de solos de cerrados no Brasil, que ocupam cerca de $20 \%$ do território nacional 
(Borgonovi et al., 1987). A baixa fertilidade deste tipo de solo é um problema complexo, e as razões para o baixo desenvolvimento das plantas ainda não são totalmente esclarecidas. Entretanto, a ocorrência de $\mathrm{Al}$ em concentrações tóxicas no solo é considerada como a principal causa deste problema em muitas situações (Blamey et al., 1992).

A calagem é uma prática cultural que possibilita minimizar este problema e que contribui para elevar o $\mathrm{pH}$, principalmente na camada arável do solo, tornando o Al menos solúvel e portanto reduzindo sua toxicidade relativa (Marion et al., 1976). Entretanto, muitas vezes esta prática cultural não é realizada, por não estar ao alcance econômico dos produtores (Pandey et al., 1994; Zeigler et al., 1995). Além disso, dada a dificuldade técnica de se realizar a calagem abaixo da camada arável, o excesso de Al trocável torna-se especialmente prejudicial no subsolo, porque reduz a profundidade e a ramificação do sistema radicular, tornando as plantas predispostas a outros tipos de estresse, como, por exemplo, a seca (Kennedy et al., 1987).

Desta forma, o desenvolvimento de materiais tolerantes ao estresse provocado pelo $\mathrm{Al}$ constitui uma alternativa ecologicamente compatível, de custo relativamente baixo, podendo proporcionar ganhos estáveis de produtividade (Duque-Vargas et al., 1994), além de reduzir os riscos da produção nestes solos (Lopes et al., 1987).

Em programas de melhoramento genético de milho visando à tolerância ao $\mathrm{Al}$, é comum a realização de seleção em solução nutritiva, contendo níveis tóxicos de $\mathrm{Al}$. Existem, entretanto, várias metodologias para avaliação da tolerância ao Al (Rhue \& Grogan, 1977; Garcia et al., 1979; Magnavaca, 1982). Principalmente no caso das gramíneas, há um consenso de que características baseadas no desenvolvimento do sistema radicular são os melhores critérios para a avaliação da tolerância.

Testando quatro diferentes tipos de índices fenotípicos para avaliação da tolerância ao $\mathrm{Al}$, Magnavaca et al. (1987a) concluíram que o comprimento relativo da raiz seminal (CRRS) foi o mais adequado para este tipo de avaliação, por ser o único a possibilitar correções quanto às diferenças de vigor que ocorrem durante a germinação. Por outro lado, Prioli (1987) verificou que o comprimento líquido da raiz seminal (CLRS) foi mais eficiente quando comparado com o CRRS, por apresentar menores coeficientes de variação.

Com o desenvolvimento da biologia molecular, tornou-se possível a utilização de modernas técnicas de marcadores moleculares, que possibilitam a identificação de regiões cromossômicas relacionadas a características quantitativas (QTLs). O uso de marcadores moleculares pode auxiliar as metodologias atualmente utilizadas em programas de melhoramento clássico, cujo objetivo é a obtenção de genótipos mais produtivos e adaptados a diferentes condições de estresse, além de permitir um melhor entendimento do controle genético destas características (Aranza et al., 1996). Na caracterização de QTLs é comum a utilização de populações segregantes, tais como $\mathrm{F}_{2}, \mathrm{~F}_{3}$ ou de retrocruzamentos, para maximizar o desequilíbrio de ligação. Em espécies que toleram alto grau de endogamia é também possível a utilização de linhagens recombinantes obtidas a partir de populações $\mathrm{F}_{2}$. Em populações $\mathrm{F}_{2}$, apesar do maior desequilíbrio de ligação, não é possível a realização de experimentos com repetições, o que pode tornar o processo de avaliação fenotípica menos preciso, devido ao pronunciado efeito de fatores ambientais (Tanksley, 1993).

Este trabalho teve por objetivo testar a eficiência dos índices fenotípicos CRRS e CLRS, na avaliação de plantas individuais de milho, para verificar a viabilidade de utilizar populações segregantes $F_{2}$ em estudos que utilizam marcadores moleculares na identificação de QTLs relacionados à tolerância ao alumínio.

\section{MATERIAL E MÉTODOS}

O experimento foi conduzido em casa de vegetação, com quatro materiais: duas linhagens endogâmicas consideradas suscetíveis ao Al, L53 e L19 (Brondani, 1993), uma linhagem endogâmica considerada tolerante (L1143) e uma variedade de polinização livre, também tolerante (CMS 36) (Parentoni, 1996). Incluiu-se, ainda, neste experimento, o fator período de germinação, com as variações de três e sete dias após a colocação das sementes para germinação. Desta forma, o experimento foi constituído de oito tratamentos, num delineamento experimental de parcelas subdivididas, com três repetições, sendo o fator período de germinação aleatorizado nas parcelas e os materiais nas subparcelas, que foram constituídas de 21 plantas. 
Em cada tratamento foram germinadas 200 sementes em papel-toalha umedecido, de maneira escalonada, para que as plantas representantes dos tratamentos no terceiro e sétimo dia de germinação estivessem aptas a serem transferidas para a solução nutritiva, em um mesmo dia Durante esta transferência, selecionaram-se as plântulas mais uniformes sem danos visíveis na raiz, medindo-se o comprimento inicial da raiz seminal (CIRS).

Estas plantas foram acondicionadas em casa de vegetação, sobre bandejas de plástico contendo 8,5 litros de solução nutritiva com $6 \mathrm{ppm}$ de $\mathrm{Al}$ na forma de $\mathrm{KAl}\left(\mathrm{SO}_{4}\right)_{2}$ (Magnavaca, 1982), sob oxigenação constante. Durante os sete dias de condução do experimento, realizaram-se, em todas as bandejas, leituras de $\mathrm{pH}$ da solução nutritiva, a cada dois dias. Pelo fato de o $\mathrm{pH}$ da solução não ter apresentado alterações maiores que 0,2 , não se realizou o ajuste de $\mathrm{pH}$. Após este período, foi realizada a medição do comprimento final da raiz seminal (CFRS).

Os dados de CIRS e CFRS foram utilizados para calcular o CLRS, que foi determinado subtraindo-se o comprimento inicial do comprimento final. Calculou-se também o CRRS, dividindo-se o CLRS pelo CIRS (Magnavaca, 1982; Prioli, 1987). Tanto o CRRS quanto o CLRS foram utilizados para a realização de análises de variância, testes de médias e distribuição de freqüências, com base nos valores fenotípicos de plantas individuais em cada um dos tratamentos avaliados.

\section{RESULTADOS E DISCUSSÃO}

Os resumos das análises de variância do experimento em parcelas subdivididas para avaliar a eficiência dos índices fenotípicos CRRS e CLRS estão apresentados na Tabela 1 .
Verificou-se, tanto para CRRS como para CLRS, que existiram efeitos significativos para genótipos (G) e para período de germinação $(\mathrm{P})$, o que indica que os materiais avaliados apresentam diferentes níveis de tolerância ao $\mathrm{Al}$ e que o tempo de germinação influenciou os índices estudados. Os coeficientes de variação para os dois índices fenotípicos avaliados foram em torno de $10 \%$, e indicam boa precisão experimental.

No caso específico do CRRS, observou-se a ocorrência de efeito significativo na interação $\mathrm{G}$ x $\mathrm{P}$, o que indica dependência entre genótipos e período de germinação, ou seja, a classificação dos genótipos quanto ao comprimento relativo de raiz seminal seria diferente dependendo do período de germinação considerado. Portanto, procedeu-se ao desdobramento da interação $P$ x G, com o intuito de estudar o comportamento dos diferentes genótipos dentro de cada período de germinação (Tabela 2). Como este tipo de análise envolve dois resíduos, foi necessário utilizar um resíduo médio e calcular o grau de liberdade deste novo quadrado médio de maneira aproximada, conforme as seguintes fórmulas (Banzatto \& Kronka, 1989):

Q.M. Res. Médio $=\frac{\text { Q.M.Res.(a)+(b-1) Q.M.Res.(b) }}{b}$

$$
\mathrm{n}^{\prime}=\frac{[\text { Q.M.Res. (a) }+(\mathrm{b}-1) \mathrm{Q} \cdot \mathrm{M} \cdot \text { Res. }(\mathrm{b})]^{2}}{\frac{[\mathrm{Q} \cdot M \cdot \text { Res.(a) }]^{2}}{\text { g.1.Res.(a) }}+\frac{[(\mathrm{b}-1) \text { Q.M.Res.(b) }]^{2}}{\text { g.1.Res.(b) }}}
$$

TABELA 1. Resumo das análises de variância empregando-se o delineamento em parcelas subdivididas para comprimento relativo de raiz seminal (CRRS) e comprimento líquido de raiz seminal (CLRS), de plântulas de milho submetidas a solução nutritiva com 6 ppm de Al tóxico. Abril de 1997.

\begin{tabular}{|c|c|c|c|}
\hline \multirow[t]{2}{*}{ Fontes de variação } & \multirow[t]{2}{*}{ G. L. } & \multicolumn{2}{|c|}{ Quadrado médio } \\
\hline & & CRRS (x 10-4) & CLRS \\
\hline Blocos & 2 & 21,53 & 0,26 \\
\hline Genótipos (G) & 3 & $5.768,91 * *$ & $103,75 * *$ \\
\hline Resíduo (a) & 6 & 101,17 & 1,28 \\
\hline Período de germinação (P) & 1 & $42.250,08 * *$ & $63,39 * *$ \\
\hline Interação G x P & 3 & $698,34 * *$ & 2,24 \\
\hline Resíduo (b) & 8 & 61,22 & 1,04 \\
\hline Total & 23 & & \\
\hline c.v. Resíduo (a) (\%) & & 12,61 & 13,18 \\
\hline c.v. Resíduo (b) (\%) & & 9,81 & 11,85 \\
\hline Média & & 0,80 & 8,60 \\
\hline
\end{tabular}


onde,

$\mathrm{a}=$ número de tratamentos principais;

$\mathrm{b}$ = número de tratamentos secundários;

n'= número de graus de liberdade aproximado do

Q. M. do resíduo médio.

Verificou-se que os genótipos apresentaram diferenças estatísticas significativas, tanto para o período de germinação aos três dias, quanto aos sete dias, indicando a necessidade de realizar testes de médias separados nas duas épocas de germinação. Os resultados do teste de médias no que tange ao CRRS, considerando os dois períodos de germinação separadamente, e do CLRS utilizando a média dos dois períodos de germinação com as respectivas variâncias amostrais, são apresentados na Tabela 3.

Observou-se, tanto aos três, como aos sete dias de germinação, que o índice fenotípico CRRS foi eficiente para discriminar as médias dos genótipos tolerantes (CMS 36 e L 1143) das médias dos genótipos suscetíveis (L 19 e L 53), o que confirma

TABELA 2. Análise de variância para o desdobramento da interação genótipo $(G) \times$ período de germinação $(\mathrm{P})$ para estudo de comprimento relativo de raiz seminal (CRRS) dentro dos períodos de germinação de três e sete dias, avaliados em plântulas de milho submetidas a solução nutritiva com 6 ppm de Al tóxico ${ }^{1}$.

\begin{tabular}{lcc}
\hline Fontes de variação & G. L. & Q. M. (x 10 ${ }^{-4}$ ) \\
\hline Genótipos dentro período de germinação (3) & 3 & $4.724,61^{* *}$ \\
Genótipos dentro período de germinação (7) & 3 & $1.742,64^{* *}$ \\
Resíduo médio & 12 & 81,19 \\
\hline
\end{tabular}

** Significativo a $1 \%$ de probabilidade pelo teste $\mathrm{F}$. os resultados relatados por Lopes et al. (1987) e Magnavaca et al. (1988).

Quando se compararam as médias de CRRS dos quatro genótipos nos dois períodos de germinação avaliados, observou-se uma inversão na classificação entre as médias dos genótipos L 1143 e CMS 36 e entre as médias dos genótipos L 19 e L 53, justificando, assim, a ocorrência de interação $\mathrm{G}$ x P significativa, apesar de os materiais não terem apresentado diferenças significativas pelo teste de Tukey $(5 \%)$. Isto mostra que os dois períodos de germinação poderiam discriminar as médias dos genótipos de maneira semelhante.

Com relação ao CLRS (Tabela 3), observou-se que este índice fenotípico também possibilitou a discriminação entre as médias dos materiais suscetíveis (L 19 e L 53) dos materiais tolerantes (CMS 36 e L 1143), possibilitando, ainda, a classificação dos materiais tolerantes em dois níveis de tolerância.

O cálculo das variâncias amostrais dos genótipos avaliados (Tabela 3) mostrou a tendência de os materiais tolerantes (L 1143 e CMS 36) apresentarem variâncias maiores em relação aos materiais suscetíveis (L 19 e L 53); isto indica que as médias dos materiais tolerantes foram estimadas com erros maiores. Este fato é influenciado pelo menos por dois fatores: o primeiro seria o efeito do Al tóxico, responsável por uma redução acentuada no desenvolvimento radicular dos materiais suscetíveis, resultando em menores médias quando comparadas aos materiais tolerantes, e o segundo seria o efeito de fatores ambientais aleatórios de difícil controle, como, por exemplo, danos físicos não visíveis no ápice da raiz seminal provocados pela manipulação das plântulas. Este efeito do ambiente pode ser

TABELA 3. Testes de médias, para o comprimento relativo de raiz seminal (CRRS) e comprimento líquido de raiz seminal (CLRS) nas avaliações realizadas aos três e sete dias de germinação com as respectivas variâncias amostrais (Vars.) para cada genótipo'.

\begin{tabular}{|c|c|c|c|c|c|c|}
\hline \multirow[t]{3}{*}{ Genótipo } & \multicolumn{4}{|c|}{ CRRS } & \multirow{2}{*}{\multicolumn{2}{|c|}{$\frac{\text { CLRS }}{\text { P. germinação }(3 \text { e } 7)}$}} \\
\hline & \multicolumn{2}{|c|}{ P. germinação (3) } & \multicolumn{2}{|c|}{ P. germinação (7) } & & \\
\hline & Média & Vars. & Média & Vars. & Média & Vars. \\
\hline L 19 & $0,834 \mathrm{~b}$ & 0,033 & $0,251 b$ & 0,014 & $6,25 c$ & 5,57 \\
\hline L 53 & $0,929 b$ & 0,075 & $0,105 b$ & 0,007 & $4,49 \mathrm{c}$ & 9,20 \\
\hline L 1143 & $1,645 \mathrm{a}$ & 0,285 & $0,535 \mathrm{a}$ & 0,050 & $9,74 b$ & 11,23 \\
\hline CMS 36 & $1,460 \mathrm{a}$ & 0,164 & $0,621 \mathrm{a}$ & 0,026 & $13,90 \mathrm{a}$ & 15,04 \\
\hline
\end{tabular}


responsável por uma redução no crescimento de raiz seminal tanto em materiais suscetíveis, como nos tolerantes de maneira semelhante. Entretanto, quando este problema afeta indivíduos suscetíveis, pelo fato de a média destes genótipos ser menor, em face do efeito do Al tóxico, estes tendem a apresentar desvios em relação à média de menor magnitude, quando comparados aos de indivíduos tolerantes.

Pelo fato de três dos materiais avaliados serem linhagens, é esperado que as 63 plantas de cada linhagem apresentem variações fenotípicas resultantes exclusivamente de efeitos ambientais. Entretanto, com relação à variedade CMS 36, além de efeitos ambientais há também variabilidade genética, por tratar-se de um material de polinização livre. Realizaram-se, então, distribuições de frequiência, tanto nos três como nos sete dias de germinação, utilizando-se o CRRS e o CLRS das 63 plantas de cada material avaliado, com o intuito de verificar o comportamento fenotípico destas plantas (Figs. 1 e 2).
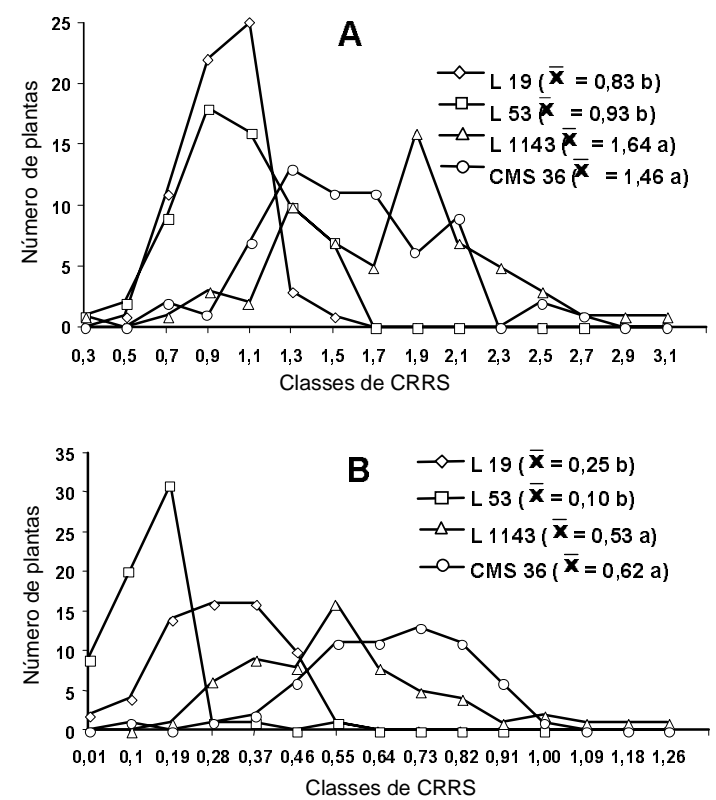

FIG. 1. Distribuição de freqüência do número de plantas nas classes de CRRS das linhagens de milho $\mathrm{L} 19$ e $\mathrm{L} 53$ suscetíveis ao $\mathrm{Al}$, da linhagem L 1143 tolerante ao Al e da variedade CMS 36 de polinização livre tolerante ao $\mathrm{Al}$, avaliadas aos três (A) e aos sete (B) dias de germinação.
Verificou-se, tanto para o CLRS como para o CRRS, a ocorrência de uma sobreposição entre os valores fenotípicos de materiais suscetíveis e tolerantes aos três e aos sete dias de germinação. No caso da avaliação para o CRRS, feita aos três dias de germinação, observou-se que $52 \%$ e $38 \%$ das plantas representantes dos materiais CMS 36 e L 1143 respectivamente, apresentaram valores de CRRS em classes de distribuição de freqüências semelhantes aos valores observados nos materiais suscetíveis. Para avaliação aos sete dias de germinação, estes valores foram $33 \%$ e $63 \%$. No CLRS, observou-se esta mesma tendência com valores de $20 \%$ e $50 \%$ para a avaliação aos três dias de germinação e $20 \%$ e $80 \%$ para avaliação aos sete dias de germinação.

Pelo fato de a sobreposição entre as curvas de distribuição de freqüências de materiais tolerantes e suscetíveis ocorrer somente no tocante aos menores valores de CRRS ou CLRS, verifica-se que em avaliações de plantas individuais a identificação de indivíduos tolerantes ao Al é mais confiável.
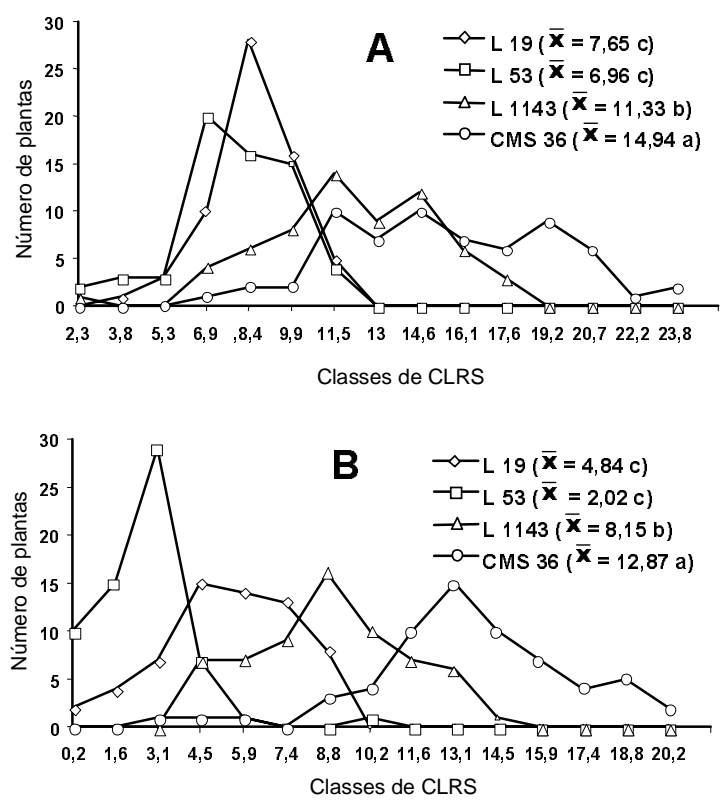

FIG. 2. Distribuição de freqüência do número de plantas nas classes de CLRS das linhagens de milho $\mathrm{L} 19$ e $\mathrm{L} 53$ suscetíveis ao $\mathrm{Al}$, da linhagem L 1143 tolerante ao Al e da variedade CMS 36 de polinização livre tolerante ao Al, avaliadas aos três (A) e aos sete (B) dias de germinação. 
A ocorrência de maior variabilidade fenotípica nos genótipos tolerantes (Tabela 3 ) e de plantas tolerantes comportando-se como suscetíveis revela a possibilidade da presença de fenômenos conhecidos como penetrância incompleta e ou expressividade variável para esta característica. Tais fenômenos, devido a fatores genéticos ou ambientais, poderiam explicar a existência de plantas tolerantes exibindo fenótipos de plantas suscetíveis, sendo responsáveis por alterações na segregação fenotípica do caráter, constituindo, assim, uma dificuldade no estudo da tolerância ao Al.

Nos dois índices fenotípicos utilizados, foram subtraídos o CIRS do CFRS, na tentativa de eliminar possíveis diferenças de vigor entre os materiais, que normalmente ocorrem durante o processo de germinação. Assim sendo, realizaram-se análises de regressão para verificar o comportamento do CRRS e do CLRS em função do CIRS, aos três e sete dias de germinação, incluindo todas as plantas dos genótipos avaliados (Figs. 3 e 4 ).
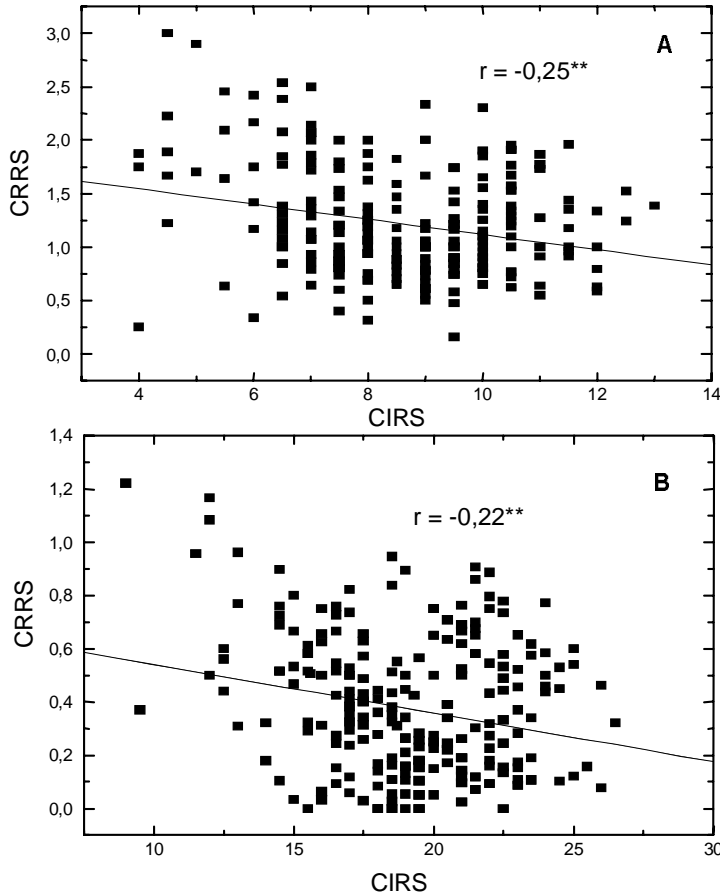

FIG. 3. Regressão entre o comprimento relativo de raiz seminal (CRRS) e o comprimento inicial de raiz seminal (CIRS) aos três (A) e sete (B) dias de germinação, para plantas dos genótipos CMS 36, L 1143, L 19 e L 53.
Verificou-se, tanto aos três como aos sete dias de germinação, que o CRRS apresentou coeficientes de correlação negativos e baixos $(r=-0,25 * *$ e $r=-0,22 * *$ aos três e sete dias de germinação, respectivamente). Isto indica que o CRRS tende a favorecer plantas que apresentam um menor CIRS no momento da transferência para a solução nutritiva. Por outro lado, para o CLRS observaram-se coeficientes de correlação positivos e baixos $(r=0,33 * *$ e $r=0,10$ aos três e sete dias de germinação, respectivamente), indicando que este índice, aos três dias de germinação, tende a favorecer plântulas com maior CIRS . Estas estimativas confirmam a tendência de coeficientes de correlação positivos em relação ao CLRS e negativos em relação ao CRRS obtidos por Magnavaca et al. (1987a), que ao realizar este mesmo tipo de abordagem relatou valores de $\mathrm{r}=-0,02$ para CRRS e $r=0,31^{* *}$ para CLRS.

Como a regressão entre CLRS e CIRS apresentou coeficiente de correlação não-significativo e a ava-
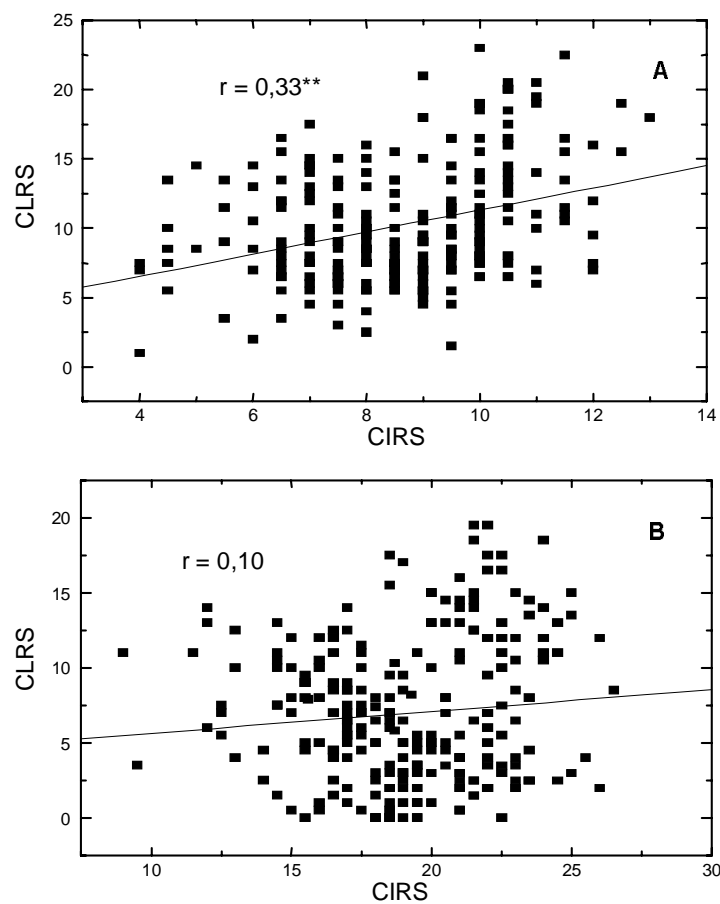

FIG. 4. Regressão entre o comprimento líquido de raiz seminal (CLRS) e o comprimento inicial de raiz seminal (CIRS) aos três (A) e sete (B) dias de germinação, para plantas dos genótipos CMS 36, L 1143, L 19 e L 53. 
liação com base no CLRS não revelou a existência de efeitos significativos para a interação genótipos (G) x período de germinação (P) (Tabela 1) e discriminou os genótipos tolerantes em duas classes distintas de tolerância (Tabela 3 ), pode-se concluir que o CLRS foi superior em relação ao CRRS para a avaliação quanto à tolerância ao $\mathrm{Al}$, quando se utilizam médias de plantas. Contudo, os resultados mostraram que tanto o CRRS como o CLRS não discriminam perfeitamente genótipos tolerantes dos suscetíveis, quando se realizam avaliações de plantas individuais. Portanto, a avaliação fenotípica de populações $\mathrm{F}_{2}$ para trabalhos de mapeamento de QTL(s), ou mesmo para estudos sobre o controle genético da tolerância ao $\mathrm{Al}$, não é a ideal, devendo-se avaliar famílias $\mathrm{F}_{3}$ e utilizar as médias destas famílias para representar os valores fenotípicos das respectivas plantas $\mathrm{F}_{2}$.

Em estudos sobre o controle genético da tolerância ao $\mathrm{Al}$, onde se utilizam populações $\mathrm{F}_{2}$ e de retrocruzamentos, ao se realizarem avaliações de plantas individuais, o fato de plantas tolerantes apresentarem valores baixos para CRRS e ou CLRS pode resultar em um viés das estimativas. Viés, este, que pode ser a causa de a tendência da suscetibilidade ao $\mathrm{Al}$ em milho ser dominante em relação à tolerância, como descrito em alguns trabalhos (Magnavaca et al., 1987b; Brondani, 1993; Torres, 1996).

\section{CONCLUSÕES}

1. Os índices fenotípicos comprimento relativo da raiz seminal e comprimento líquido da raiz seminal são eficientes na avaliação de genótipos de milho quanto à tolerância ao Al, quando se utilizam médias de vários indivíduos.

2. A utilização do comprimento relativo da raiz seminal ou comprimento líquido da raiz seminal na avaliação de plantas individuais referente à tolerância ao Al está sujeita a erros significativos, e não possibilita uma discriminação confiável, principalmente de indivíduos suscetíveis.

3. A existência de plantas tolerantes com valores fenotípicos característicos de plantas suscetíveis devido ao efeito genético ou ambiental, provoca um viés de estimativas, que pode resultar em tendência de a suscetibilidade ser dominante em relação à tolerância ao $\mathrm{Al}$.

4. Em estudos de mapeamento de QTLs empregando-se marcadores moleculares, e em trabalhos sobre o controle genético da tolerância ao $\mathrm{Al}$, deve-se realizar a avaliação fenotípica de famílias $\mathrm{F}_{3}$, e utilizar suas médias para representar os valores fenotípicos das respectivas plantas $F_{2}$.

\section{AGRADECIMENTOS}

Ao Conselho Nacional de Desenvolvimento Científico e Tecnológico $(\mathrm{CNPq})$, à Fundação de Amparo à Pesquisa do Estado de Minas Gerais (FAPEMIG) e à Agência Internacional de Energia Atômica, pelo suporte financeiro; aos técnicos Miguel F. A. dos Reis e Gislene R. B. Cristeli, pela colaboração na condução do experimento.

\section{REFERÊNCIAS}

ARANZA, F.; TADMOR, Y.; KLEIN, B.P.; ROCHEFORD, T.R.; JUVIK, J.A. Quantitative trait loci influencing chemical and sensory characteristics of eating quality in sweet corn. Genome, v.39, p.40-50, 1996

BANZATTO, D.A.; KRONKA, S. do N. Experimentação agrícola. Jaboticabal: FUNEP, 1989. 247p.

BLAMEY, F.P.C.; ROBINSON, N.J.; ASHER, C.J. Interspecific differences in aluminium tolerance in relation to root cation-exchange capacity. Plant and Soil, v.146, p.77-82, 1992.

BORGONOVI, R.A.; SCHAFFERT, R.E.; PITTA, G.V.E.; MAGNAVACA, R.; ALVES, V.M.C. Aluminum tolerance in sorghum. In: GABELMAN, H.W.; LOUGNMAN, B.C. (Eds.). Genetics aspects of plant mineral nutrition. Dordrecht: Martinus Nijhoff, 1987. p.213-221

BRONDANI, C. Análise de RFLP no estudo da toxidez do alumínio em milho. Lavras: ESAL, 1993. 78p. Tese de Mestrado

DUQUE-VARGAS, J.; PANDEY, X.; GRANADOS, G.; CEBALLOS, H.; KNAPP, E. Inheritance of tolerance to soil acidity in tropical maize. Crop Science, v.34, p.50-54, Jan./Feb. 1994

Pesq. agropec. bras., Brasília, v.34, n.10, p.1897-1904, out. 1999 
GARCIA, O.; SILVA, W.J.; MASSEI, M.A.S. An efficient method for screening maize inbreds for aluminum tolerance. Maydica, Bergamo, v.24, n.2, p.75-82, Apr./Jun. 1979.

KENNEDY, C.W.; BA, M.T.; CALDWELL, A.G.; HUTCHINSON, R.L.; JONES, J.E. Differences in root and shoot growth and soil moisture extraction between cotton cultivars in an acid subsoil. Plant and Soil, v.101, p.241-246, 1987.

LOPES, M.A.; MAGNAVACA, R.; BAHIA FILHO, A.F.C.; GAMA, E.E.G. e. Avaliação de populações de milho e seus cruzamentos para tolerância à toxidez de alumínio em solução nutritiva. Pesquisa Agropecuária Brasileira, Brasília, v.22, n.3, p.257263, mar. 1987

MAGNAVACA, R. Genetic variability and inheritance of aluminum tolerance in maize. Lincoln: Univ. of Nebraska, 1982. 135p. Ph.D. Thesis.

MAGNAVACA, R.; GAMA, E.E.G. e; BAHIA FILHO, A.F.C.; FERNANDES, F.T. Obtenção de híbridos duplos de milho para tolerância a toxidez de alumínio. Pesquisa Agropecuária Brasileira, Brasília, v.23, n.9, p.971-977, set. 1988.

MAGNAVACA, R.; GARDNER, C.O.; CLARK, R.B. Evaluation of inbred maize lines for aluminum tolerance in nutrient solution. In: GABELMAN, H.W.; LOUGHMAN, B.C. (Eds.). Genetic aspects of plant mineral nutrition. Dordrecht: Martinus Nijhoff, 1987a. p.255-265.

MAGNAVACA, R.; GARDNER, C.O.; CLARK, R.B Inheritance of aluminum tolerance in maize. In: GABELMAN, W.H.; LOUGHMAN, B.C. (Eds.) Genetic aspects of plant mineral nutrition Dordrecht: Martinus Nijhoff, 1987b. p.201-211.
MARION, G.M.; HENDRICKS, D.M.; DULL, G.R.; FULLER, W.H. Aluminum and silica solubility in soils. Soil Science, v.121, p.76-85,1976.

PANDEY, S.; CEBALLOS, H.; MAGNAVACA, R.; BAHÍA FILHO, A.F.C.; DUQUE-VARGAS, J.; VINASCO, L.E. Genetics of tolerance to soil Acidity in tropical maize. Crop Science, v.34, p.1511$1514,1994$.

PARENTONI, S.N. Avanços no programa de adaptação de milho a solos ácidos do CNPMS/Embrapa In: REUNIÓN DE COORDINADORES SURAMERICANOS DE PROGRAMAS DE MAIZ, 4., 1996, Cali. Memorias. Cali: CIMMYT, 1996. p.74-103.

PRIOLI, A.J. Análise genética da tolerância à toxidez do alumínio em milho (Zea mays L.). Campinas: UNICAMP, 1987. 182p. Tese de Doutorado.

RHUE, R.D.; GROGAN, C.O. Screening corn for Al tolerance using different $\mathrm{Ca}$ e $\mathrm{Mg}$ concentration Agronomy Journal, Madison, v.69, n.5, p.755-760, Sept./Oct. 1977.

TANKSLEY, S.D. Mapping polygenes. Annual Review of Genetics, v.27, p.205-233, 1993

TORRES, G.A. Identificação de regiões cromossômicas do milho associadas com tolerância ao alumínio tóxico por meio de RFLP. Lavras: UFLA, 1996. 62p. Tese de Mestrado.

ZEIGLER, R.S.; PANDEY, S.; MILES, J.; GOURLEY, L.M.; SARKARUNG, S. Advances in the selection and breeding of acid-tolerant plants: rice, maize sorghum and tropical forages. In: DATE, R.A.; GRUNDON, N.J.; RAYMENT, G.E.; PROBERT, M.E. (Eds.). Plant-soil interactions at low pH: principles and management. Dordrecht: Kluwer Academic, 1995. p.391-406. 